\title{
Caracterización y modificación de halloysitas naturales para la adsorción de contaminantes emergentes. Revalorización de recursos naturales.
}

\section{Characterization and modification of natural halloysites for the adsorption of emerging pollutants. Revaluation of natural resources.}

Presentación: 6 y 7 de octubre de 2020

\section{Doctorando:}

\section{Martina Ormaechea}

Centro de Tecnología de Recursos Minerales y Cerámica (CETMIC), Argentina.

ormaecheamartina@gmail.com

\section{Director/a:}

\section{Mariela A. Fernández}

\section{Resumen}

Los contaminantes emergentes (CE) son compuestos de distinto origen y naturaleza química. Productos farmacéuticos, productos de cuidado personal, detergentes, subproductos de desinfección, medicamentos y retardantes de llama, son algunos ejemplos de CE. Debido a su presencia en agua, los efectos ecotoxicológicos que producen y las características bioacumulativas, pueden influir en la biota acuática y por ende en la salud humana.

Las propiedades de las halloysitas (Hall) y su bajo costo, permiten plantearlas como adsorbentes de estas sustancias en agua. En particular, sus derivados tubulares, presentan una ventaja para la construcción de lechos de columnas filtrantes. Además, sus superficies tanto interna como externa pueden ser fácilmente modificadas con tratamientos químicos diversos. Estas modificaciones les permiten ampliar sus propiedades adsortivas.

En este trabajo se emplearon dos halloysitas naturales (muestra A: 90\% Hall tubular y muestra B: 40/60\% Hall esferoidal/tubular). Los depósitos de estas arcillas están ubicados en el área de Mamil Choique y Buitrera, Patagonia, Argentina; particularmente se encuentran formando agregados donde conviven formas esferoidales y tubulares.

Las muestras de Hall se modificaron mediante tratamiento ácido $\left(\mathrm{H}_{2} \mathrm{SO}_{4} 0.1 \mathrm{M}\right)$, alcalino $(\mathrm{KOH} 0.1 \mathrm{M})$ y tratamiento térmico (400 y $800^{\circ} \mathrm{C}$ ). Las Hall y sus modificaciones fueron caracterizadas mediante difracción de rayos X (DRX), potencial zeta (PZ) y microscopia electrónica de barrido (MEB).

Se pretende optimizar la modificación de las Hall para luego evaluar su posible uso en la adsorción de contaminantes emergentes. 
Palabras clave: halloysitas, adsorción, contaminantes emergentes

\begin{abstract}
Emerging pollutants (EC) are compuonds of different origin and chemical nature. Pharmaceuticals, personal care products, detergents, desinfection by-products, drugs, and flams retardants are come examples of EC. Due to their presence in water, the ecotoxicological effects they produce and the bioaccumulative characteristics, can influence aquatic biota and therefore human health.
\end{abstract}

The properties of halloysites (Hall) and their low cost, allow them to be considered as adsorbents for these substances in water. In particular, its tubular derivatives have an advantage for the construction of filter column beds. In addition, its internal and external surfaces can be easily modified with various chemical treatments. These modifications allow them to expand their adsorptive properties.

In this work, two natural halloysites were used (sample A: 90\% Hall tubular and sample B: 40/60\% Hall spheroidal / tubular). The deposits of these clays are located in the area of Mamil Choique and Buitrera, Patagonia, Argentina; particularly they are found forming aggregates where spheroidal and tubular forms coexist.

The Hall samples were modified by acid treatment $(0.1 \mathrm{M} \mathrm{H} 2 \mathrm{SO} 4)$, alkaline $(0.1 \mathrm{M} \mathrm{KOH})$ and heat treatment $\left(400\right.$ and $800^{\circ}$ C). Halls and their modifications were characterized by X-ray diffraction (XRD), zeta potential (PZ) and scanning electron microscopy (SEM).

It is intended to optimize the modification of the Halls and then evaluate their possible use in the adsorption of emerging pollutants.

Keywords: halloysite, adsorption, emerging pollutants

\title{
Introducción
}

Los contaminantes emergentes (CE) son compuestos de distinto origen y naturaleza química que no se consideran abundantes en el medio ambiente. No obstante, se caracterizan por su persistencia en agua y su amenaza en la salud ambiental (Peña-Guzmán et al, 2019, Miniero et al 2014). Pesticidas, productos farmacéuticos, productos de cuidado personal, detergentes, subproductos de desinfección, medicamentos y retardantes de llama, son algunos ejemplos de CE. Debido a su presencia en agua, los efectos ecotoxicológicos que producen y las características bioacumulativas, pueden influir en la biota acuática y por ende en la salud humana.

Los minerales arcillosos laminares son adsorbentes por excelencia, sin embargo, su aplicación tecnológica en el desarrollo de columnas de remediación está limitada por su gran capacidad de hinchamiento que produce la obstrucción del sistema (Low 1980). Los materiales fibrosos o tubulares de naturaleza mineral ya sea naturales o sintéticos se encuentran en auge ya que presentan aplicaciones variables y son versátiles para su utilización como materiales de relleno en columnas de purificación de aguas ya que su estructura particular presenta ventajas frente a los minerales arcillosos de estructura laminar tradicionales y/o nanotubos sintéticos como los nanotubos de carbono (Yuan et al 2015). Los minerales naturales con morfología tubular o fibrosa son provenientes de las familias de caolinitas y de las sepiolitas. Particularmente de la familia de las caolinitas se encuentra un grupo de arcillas con morfologías de naturaleza tubular, placas o esférica, las halloysitas (Cravero et al 2012). Las cuales poseen 
la fórmula empírica $\mathrm{Al}_{2} \mathrm{Si}_{2} \mathrm{O}_{5}(\mathrm{OH})$. La particularidad de la halloysita radica en su estructura y morfología. Específicamente, tiene moléculas de agua entre las capas y dependiendo del espacio que ocupa el agua hay dos formas del mineral, el hidratado con espaciamiento basal cercano a $1 \mathrm{~nm}$ llamado "halloysite- $(10 \AA)$ " y el deshidratado con $0,72 \mathrm{~nm}$, espacio conocido como "halloysite- (7 Å)" (Ferrante et al, 2015). Las características de los nanocristales de halloysita varían según la génesis y las condiciones de formación. La longitud típica de los nanotubos es de 0.02 a $30 \mu \mathrm{m}$ y los diámetros varían entre 0.05 y $0.2 \mu \mathrm{m}$ (Li et al 2019). La carga de la parte interior de las nanopartículas es positiva mientras que la carga de la superficie exterior es negativa. Esto es causado por la disposición de elementos químicos de la halloysita. La superficie exterior está estructurada por una lámina tetraédrica $\left(\mathrm{SiO}_{2}\right)$ y la interna está estructurada por una lámina octaédrica $\left(\mathrm{Al}(\mathrm{OH})_{3}\right)$.

Los mayores depósitos conocidos de halloysita se han encontrado en los Estados Unidos, China, Turquía, Nueva Zelanda, Francia y Bélgica. (Wang and Wang 2017). En Argentina, la halloysita se encuentra en algunas áreas. Por ejemplo, se encuentran yacimientos de halloysita, caolinita y sericita, junto con cristobalita y sanidina, en La Buitrera I y II de la provincia de Río Negro y que el tipo de mineralización se puede encontrar en la misma provincia en el área de Mamil Choique. (Cravero et al. 2009). Particularmente los yacimientos de halloysitas en Argentina se encuentran formando agregados donde conviven formas esferoidales y tubulares con longitudes menores a $0.2 \mu \mathrm{m}$ (Cravero and Churchman, 2016). Debido a la diferente distribución de carga en el tubo de halloysita, este mineral puede interactuar con diferentes moléculas e iones. No solo se puede usar halloysita pura sino también modificada, para el atrapamiento de una amplia variedad de moléculas (Anastolopoulus et al, 2018).

Describir las modificaciones posibles, térmica, mecánica que pueden ampliar sus capacidades.

\section{Desarrollo}

Se emplearon dos halloysitas (muestra A: 90\% Hall tubular y muestra B: 40/60\% Hall esferoidal/tubular). Los depósitos de estas arcillas están ubicados en el área de Mamil Choique y Buitrera, Patagonia, Argentina.

- Modificación Química.

- Tratamiento ácido $\left(\mathrm{H}_{2} \mathrm{SO}_{4} 0.1 \mathrm{M}\right)$,

- Tratamiento alcalino (KOH $0.1 \mathrm{M})$

- Tratamiento térmico $\left(400\right.$ y $\left.800^{\circ} \mathrm{C}\right)$.

- Caracterización de Halloysitas.

- Difracción de rayos X (DRX),

- Potencial zeta (PZ) y

- Microscopia electrónica de barrido (MEB).

\section{Resultados}

- $\quad$ Microscopia electrónica de barrido (MEB)

La siguiente imagen SEM muestra la presencia de tubos cortos de halloysita de $2.5 \mu \mathrm{m}$ de largo correspondientes a la muestra A. 


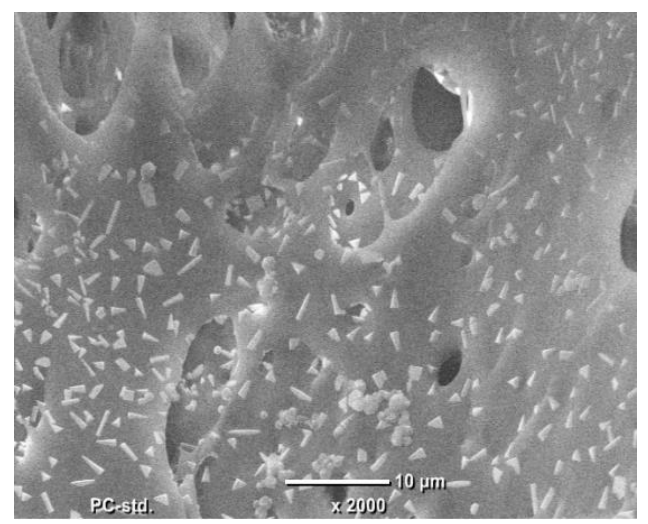

Figura 1. Micrografía electrónica de halloysitas tubulares.

- Potencial Zeta (PZ)
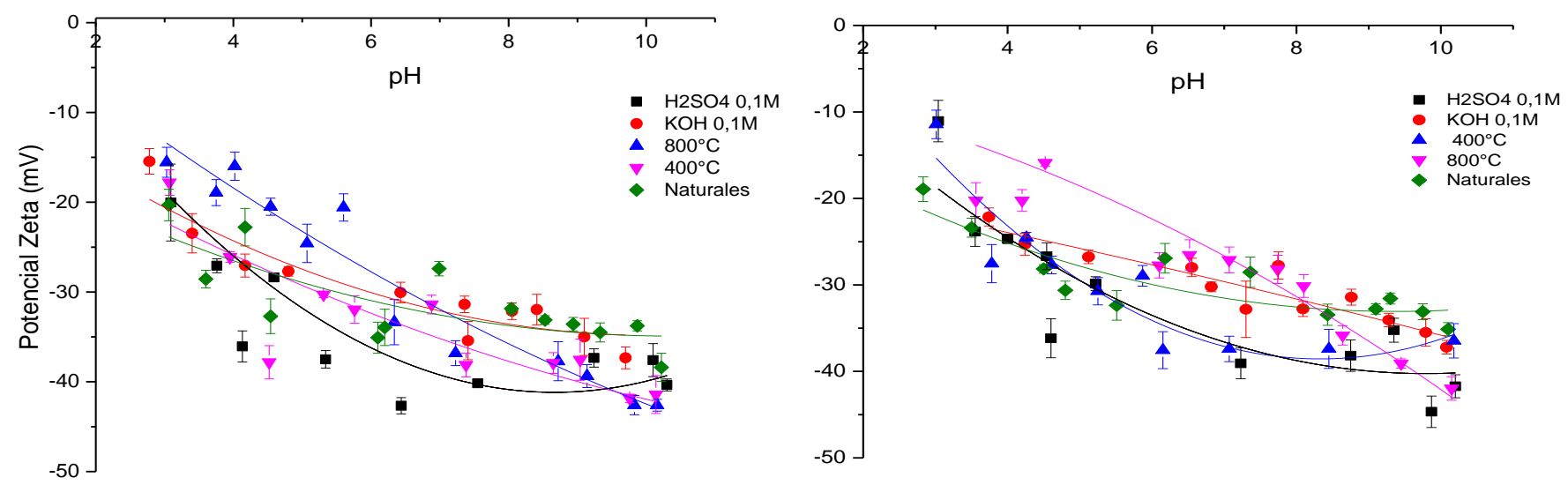

Figura 2. Carga superficial de hall (tipo A izq. y tipo B der.).

Los valores obtenidos fueron negativos debido a la presencia de grupos siloxano y silanol en la superficie externa. Sin embargo, no se observan grandes cambios superficiales.

- Difracción de rayos X

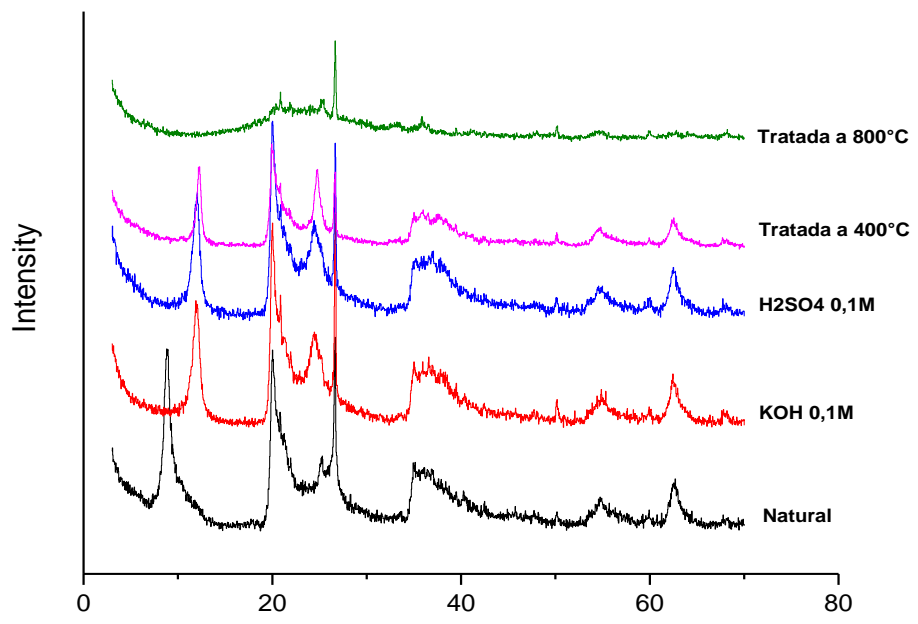

Pos. [ ${ }^{\circ}$ Th.]

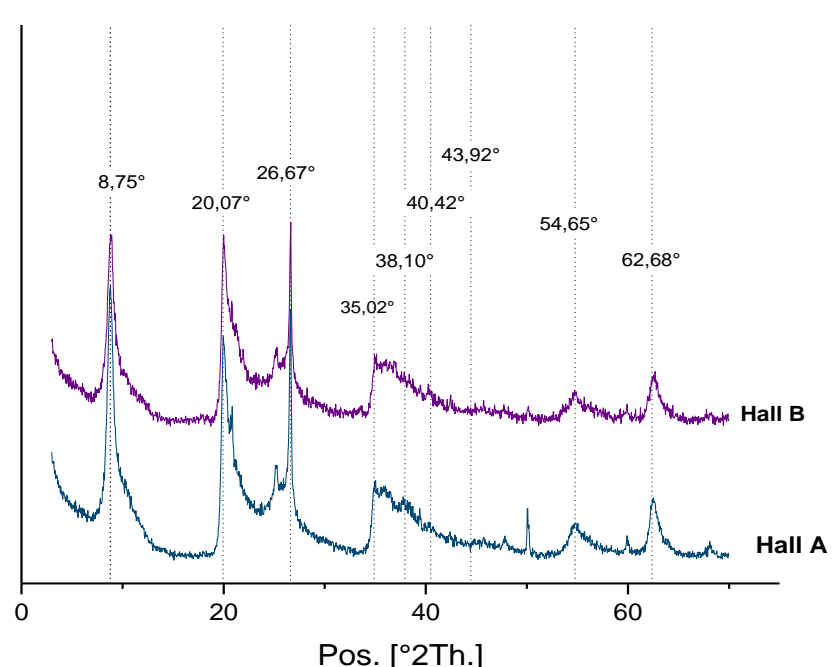

Pos. [02Th.] 
Figura 3. Análisis de DRX que muestran las fases correspondientes a halloysita (der). Solo se observa pérdida de dicha estructura con el tratamiento térmico (izq.).

Se puede describir los cambios observados en los picos. Con respecto a las muestras naturales.

Las muestras tratadas a 800 se observa perdida de estructura desaparecen los picos correspondientes a las fases de halloysitas. Después el tratamiento ácido y alcalino también producen cambios. Ver el pico a 8.75 que distancia en el plano representa porque ese es el que ataca. Achica ese espaciado.

\section{Conclusiones}

- Las superficies de las Hall tanto interna como externa pueden ser modificadas con tratamientos químicos diversos.

- Solo se observó un cambio en la estructura de ambas halloysitas luego de realizar tratamiento térmico.

- Se pretende optimizar la modificación de las Hall para luego evaluar su posible uso en la adsorción de contaminantes emergentes.

\section{Referencias}

Anastopoulos I, Mittal A., Usman M. Mittal J, Yue G, Avelino Núñez-Delgado A. Kornaros M. A review on halloysitebased adsorbents to remove pollutants in water and wastewater. Journal of Molecular Liquids. Volume 269, 1 November 2018, Pages 855-868.

Awad A. M., Shaikh S. M.R., Jalab R. Gulied M. H. Nasser Benamor M.S. Adham A. S. Adsorption of organic pollutants by natural and modified clays: A comprehensive review. Separation and Purification Technology Volume 228, 1 December 2019, 115719

Bianchi A, Fernández M., Torres Sánchez. R. Appl. Clay Sci. 2013,83, 280-285.

Chen, J.Y., Li, G.Y., He, Z.G., An, T. J. Hazard. Mater. 190, 416-423, 2011

Cravero F, and Churchman G. J. The origin of spheroidal halloysites: a review of the literature Clay Minerals, (2016) 51, $417-427$

Cravero F, Marfil S. A, Ramos C. P. and Maiza P. Coexistence of halloysite and iron-bearing clays in an altered ignimbrite, Patagonia, Argentina. Clay Minerals, (2014) 49, 429-441.

Cravero F. Maiza P. J, and Marfil S. A. Halloysite in Argentinian deposits: origin and textural constraints. Clay Minerals, (2012) 47, 329-340

Cravero F.; Martinez A.; Pestalardo F. Yacimientos de halloysita en la zona de Mamil Choique, Provincia de Río Negro, Patagonia, Argentina. Revista de la Asociación Geológica Argentina; Lugar: Buenos Aires; Año: 2009 vol. 65 p. 586 - 592

Damonte M. Torres Sánchez R. M., dos Santos M. Appl. Clay Sci. 26, 86-94.,2007 
Deng L, Yuan P, Liu D., Bergaya F, Zhou J, Chen F, Liu Z. Effects of microstructure of clay minerals, montmorillonite, kaolinite and halloysite, on their benzene adsorption behaviors. Applied Clay Science. Volume 143, July 2017, Pages 184191

Farmer V. C., Adams M. J., Fraser A. R. and Palmieri F. Synthetic Imogolite: Properties, Synthesis, And Possible Applications. Clay Minerals (1983) 18, 459-472.

Fernández M, Curutchet G., Torres Sánchez R. Water, Air, Soil Poll. 2014, 225:1987-1998.

Ferrante F, Armata N, and Lazzara G. Modeling of the Halloysite Spiral Nanotube. DOI: 10.1021/acs.jpcc.5b04281. J. Phys. Chem. C 2015, 119, 16700-16707

Guz L., Curutchet G., Torres Sánchez R.M. J. Environ. Chem. 2014, 2: 2344-2351.

Lescano L, Marfil S, Maiza P, Sfragulla J, Bonalumi A. HALLAZGO DE SEPIOLITA EN SERPENTINITAS, MINA ÁRBOL SECO, PROVINCIA DE CÓRDOBA. Revista de la Asociación geológica argentina. Vol 69, No 4 (2012)

Lombardi B, Baschini M., Torres Sánchez R. Appl. Clay Sci. 22, 309-312, 2003.

Lombardi B, Torres Sánchez R. M., Eloy P., et al. Appl. Clay Sci. 33, 59-65, 2006.

Low, P.F.: The swelling of clay: II. Montmorillonites. Soil Sci. Soc. Am. 46, 667-676 (1980)

Miniero R, Abate V, Brambilla G, Davoli E., De Felip E., De Filippis S.P., Dellatte E., De Luca S., Fanelli R., Fattore E., Ferri F., Fochi I., Fulgenzi A., Iacovella N., Iamiceli A.L., Lucchetti D., Melotti P., Moret I., Piazza R., Roncarati A., Ubaldi A., Zambon S., di Domenico A. Persistent toxic substances in Mediterranean aquatic species Sci. Total Environ., 494-495 (2014), pp. 18-27, 10.1016/j.scitotenv.2014.05.131

Park D-H, Jang M. W., Shul Y-G, and Choy J-H. Sepiocite, Sepiolite-Like Nanoclay Derived from Hydrotalcite-Like Layered Double Hydroxide Journal of Nanoscience and Nanotechnology Vol. 11, 382-385, 2011

Peña-Guzmán C., Ullo-Sánchez S, Mora K, Bustos R.H, Lopez-Barrera E, Alvarez J, Rodriguez-Pinzón M. Emerging pollutants in the urban water cycle in Latin America: A review of the current literatura. Journal of Environmental Management Volume 237,1 May 2019, Pages 408-423

Robson. Synthetic halloysites Apr. 17, 1979.

Tribe L, Khoury G, Torres Sánchez., R., dos Santos M. Apl. Clay Sci, 50, 167-175, 2010.

Wang W and Wang A. Nanoscale Clay Minerals for Functional Ecomaterials: Fabrication, Applications, and Future Trends. 2017

Wu, P.X., Liao, Z.W., Zhang, H., Guo, J. Environ. Inter. 26, 401-407, 2001.

Yuan P, Tan D, -Bergaya F.Properties and applications of halloysite nanotubes: recent research advances and future prospects. Applied Clay Science 112-113 (2015) 75-93. 